\title{
Crystal structure of $\eta^{1}$-benzoato-chloro-tris(1-pyrazolylmethane)- copper(II), $\mathrm{C}_{17} \mathrm{H}_{15} \mathrm{ClCuN}_{6} \mathrm{O}_{2}$
}

\author{
K. A. Van Langenberg', B. Moubaraki', K. S. Murray ${ }^{\mathrm{I}}$ and E. R. T. Tiekink*,II,I \\ I Monash University, School of Chemistry, PO Box 23, Clayton, Victoria 3800, Australia \\ IT The University of Adelaide, Department of Chemistry, Australia 5005
}

Received March 25, 2002, accepted and available on-line May 10, 2002; CCDC-No. 1267/833

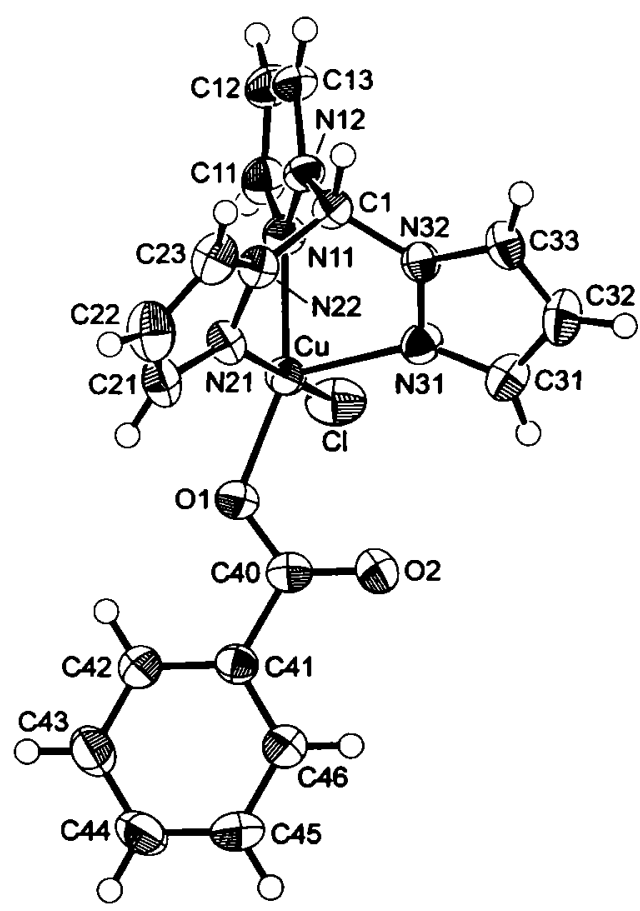

\section{Abstract}

$\mathrm{C}_{17} \mathrm{H}_{15} \mathrm{ClCuN} 6 \mathrm{O}_{2}$, monoclinic, $P 121 / c 1$ (No. 14), $a=7.715(3) \AA$, $b=14.382(2) \AA, c=16.457(3) \AA, \beta=99.95(2)^{\circ}, V=1798.6 \AA^{3}$, $Z=4, R_{\mathrm{gt}}(F)=0.041, w R_{\mathrm{ref}}\left(F^{2}\right)=0.110, T=293 \mathrm{~K}$.

\section{Source of material}

The $\mathrm{Cu}$ (II) complex was prepared using the method reported in [1] in which (tris-pyrazolylmethane) $\mathrm{CuCl}_{2}$ was reacted with an equimolar amount of sodium benzoate in methanol. Light blue crystals formed upon evaporation of the solvent

\section{Discussion}

The $\mathrm{Cu}$ atom exists in a five coordinate environment defined by a chloride, $d(\mathrm{Cu}-\mathrm{Cl}) 2.2486(10) \AA, \mathrm{O} 1$ from a monodentate benzoate, $d(\mathrm{Cu}-01,02) 1.909$ (2) $\AA$ and 3.080(2) $\AA$, and nitrogen atoms derived from the tridentate ligand. The coordination polyhedron is best described as been based on a distorted square pyramid. In this description the deviations of the $\mathrm{Cl}, 01, \mathrm{~N} 11$ \& N21 atoms from their least-squares plane are $0.103(1) \AA,-0.115(2) \AA$, -0.114 (3) $\AA$ and $0.126(3) \AA$, respectively, and the $\mathrm{Cu}$ atom lies 0.2348 (3) A out of this plane in the direction of the N31 atom. The alternative description is one based on a trigonal bipyramid for which the $\mathrm{Cl}-\mathrm{Cu}-\mathrm{N} 21$ angle is $172.86(8)^{\circ}$. The $\mathrm{Cu}-\mathrm{N}$ distances

* Correspondence author (e-mail: chmtert@nus.edu.sg)

1 Current address: National University of Singapore, Department of Chemistry, Singapore 117543 for the nitrogen atoms defining the basal plane of 2.033(3) $\AA$ and $2.069(3) \AA$ are significantly shorter than apical $d(\mathrm{Cu}-\mathrm{N} 31)$ of 2.274(3) $\AA$. In the crystal structure there are both $\mathrm{C}-\mathrm{H} \cdots \pi$ and $\mathrm{C}-\mathrm{H} \cdots \mathrm{O}$ interactions of note. The most significant $\mathrm{C}-\mathrm{H} \cdots \pi$ contacts involve the $\mathrm{C12}-\mathrm{H}$ atom which is orientated towards a cleft defined by translationally (along the $a$-axis) related N21-N22 and N31-N32 rings so that $d(\mathrm{H}$-..ring centroid of N31-N32) $=2.87 \AA$ and the angle at $\mathrm{H}=134^{\circ}$. The parameters involving the N21-N22 ring are $2.95 \AA$ and $131^{\circ}$. The $\mathrm{O} 2$ atom forms two close contacts with a neighbouring molecule so that $d\left(\mathrm{C} 1-\mathrm{H} \cdots \mathrm{O} 2^{i}\right)=2.20 \AA$, $d\left(\mathrm{C} 1 \cdots 02^{i}\right)=3.102(4) \mathrm{A}$ and the angle at $\mathrm{H}$ is $152^{\circ}$; symmetry operation $i$ : $1-x, 1 / 2+y,-1 / 2-z$. The other contact involves $C 13-H$ and has parameters associated with it of $2.47 \AA, 3.155(5) \AA$ and $131^{\circ}$, respectively. These complexes have been used in studies [2] of bioinorganic modelling of copper proteins such as hemocyanin in a related way to the tris-pyrazolylhydridoborate analogues [3] and the Cu-tacn (triazacyclononane) [4] compounds studied in $[3,4]$.

Table 1. Data collection and handling.

\begin{tabular}{ll}
\hline Crystal: & blue block, size $0.16 \times 0.21 \times 0.24 \mathrm{~mm}$ \\
Wavelength: & Mo $K_{a}$ radiation $(0.71073 \AA)$ \\
$\mu:$ & $13.89 \mathrm{~cm}^{-1}$ \\
Diffractometer, scan mode: & Rigaku AFC6R, $\omega / 2 \theta$ \\
$2 \theta_{\text {max: }}$ & $55.2^{\circ}$ \\
$N(\text { hll })_{\text {measured, }} N(h k l)_{\text {mnique }}:$ & 4626,4147 \\
Criterion for $I_{\text {obs }}, N(h k l)_{\mathrm{gt}}:$ & $I_{\text {obs }}>2 \sigma\left(I_{\text {obs }}\right), 2780$ \\
$N(\text { param })_{\text {refined: }}$ & 245 \\
Programs: & SIR92 [5], teXsan [6], SHELXL-97 [7], \\
& PLATON [8], DIBABS [9], ORTEPII [10]
\end{tabular}

Table 2. Atomic coordinates and displacement parameters (in $\AA^{2}$ ).

\begin{tabular}{|c|c|c|c|c|c|}
\hline Atom & Site & $x$ & $y$ & $z$ & $U_{\text {iso }}$ \\
\hline $\mathbf{H}(1)$ & $4 e$ & 0.5967 & -0.3626 & -0.2424 & $0.061(3)$ \\
\hline $\mathbf{H}(11)$ & $4 e$ & 0.9879 & -0.6051 & -0.3362 & 0.061 \\
\hline$H(12)$ & $4 e$ & 1.1508 & -0.4649 & -0.2797 & 0.061 \\
\hline$H(13)$ & $4 e$ & 0.9332 & -0.3570 & -0.2380 & 0.061 \\
\hline$H(21)$ & $4 e$ & 0.2695 & -0.5075 & -0.5008 & 0.061 \\
\hline $\mathrm{H}(22)$ & $4 e$ & 0.1978 & -0.3406 & -0.4891 & 0.061 \\
\hline H(23) & $4 e$ & 0.3703 & -0.2865 & -0.3553 & 0.061 \\
\hline$H(31)$ & $4 e$ & 0.3615 & -0.6710 & -0.1698 & 0.061 \\
\hline H(32) & $4 e$ & 0.3638 & -0.5543 & -0.0585 & 0.061 \\
\hline H(33) & $4 e$ & 0.4889 & -0.4099 & -0.1069 & 0.061 \\
\hline$H(42)$ & $4 e$ & 0.2178 & -0.7146 & -0.5699 & 0.061 \\
\hline$H(43)$ & $4 e$ & 0.0221 & -0.7806 & -0.6758 & 0.061 \\
\hline$H(44)$ & $4 e$ & -0.1775 & -0.8889 & -0.6477 & 0.061 \\
\hline$H(45)$ & $4 e$ & -0.1876 & -0.9277 & -0.5133 & 0.061 \\
\hline$H(46)$ & $4 e$ & 0.004 & -0.8589 & -0.4065 & 0.061 \\
\hline
\end{tabular}


Table 3. Atomic coordinates and displacement parameters (in $\AA^{2}$ ).

\begin{tabular}{|c|c|c|c|c|c|c|c|c|c|c|}
\hline Atom & Site & $x$ & $y$ & $z$ & $U_{11}$ & $U_{22}$ & $U_{33}$ & $U_{12}$ & $U_{13}$ & $U_{23}$ \\
\hline $\mathrm{Cu}$ & $4 e$ & $0.54909(5)$ & $-0.62112(3)$ & $-0.35358(2)$ & $0.0332(2)$ & $0.0344(2)$ & $0.0327(2)$ & $-0.0022(2)$ & $0.0046(1)$ & $-0.0026(2)$ \\
\hline $\mathrm{Cl}(1)$ & $4 e$ & $0.7033(1)$ & $-0.75242(6)$ & $-0.32029(7)$ & $0.0491(5)$ & $0.0373(4)$ & $0.0727(7)$ & $0.0049(4)$ & $0.0051(4)$ & $-0.0033(4)$ \\
\hline$O(1)$ & $4 e$ & $0.3652(3)$ & $-0.6789(2)$ & $-0.4293(1)$ & $0.049(1)$ & $0.049(1)$ & $0.039(1)$ & $-0.016(1)$ & $-0.002(1)$ & $0.002(1)$ \\
\hline$O(2)$ & $4 e$ & $0.2487(3)$ & $-0.7561(2)$ & $-0.3346(1)$ & $0.048(1)$ & $0.061(2)$ & $0.035(1)$ & $-0.009(1)$ & $0.003(1)$ & $0.001(1)$ \\
\hline$N(11)$ & $4 e$ & $0.7672(3)$ & $-0.5431(2)$ & $-0.3143(2)$ & $0.031(1)$ & $0.037(1)$ & $0.038(1)$ & $-0.000(1)$ & $0.009(1)$ & $-0.003(1)$ \\
\hline$N(12)$ & $4 e$ & $0.7527(3)$ & $-0.4577(2)$ & $-0.2802(2)$ & $0.028(1)$ & $0.036(1)$ & $0.036(1)$ & $-0.004(1)$ & $0.007(1)$ & $-0.001(1)$ \\
\hline$N(21)$ & $4 e$ & $0.4313(3)$ & $-0.4959(2)$ & $-0.3926(2)$ & $0.038(2)$ & $0.042(2)$ & $0.029(1)$ & $0.003(1)$ & $0.003(1)$ & $-0.002(1)$ \\
\hline $\mathbf{N}(22)$ & $4 e$ & $0.4637(3)$ & $-0.4195(2)$ & $-0.3441(2)$ & $0.032(1)$ & $0.035(1)$ & $0.035(1)$ & $0.003(1)$ & $0.007(1)$ & $0.002(1)$ \\
\hline $\mathbf{N}(31)$ & $4 e$ & $0.4703(3)$ & $-0.5750(2)$ & $-0.2329(2)$ & $0.037(1)$ & $0.034(1)$ & $0.038(2)$ & $-0.001(1)$ & $0.015(1)$ & $-0.002(1)$ \\
\hline$N(32)$ & $4 e$ & $0.5127(3)$ & $-0.4860(2)$ & $-0.2100(2)$ & $0.035(1)$ & $0.034(1)$ & $0.030(1)$ & $0.000(1)$ & $0.009(1)$ & $-0.004(1)$ \\
\hline$C(1)$ & $4 e$ & $0.5825(4)$ & $-0.4250(2)$ & $-0.2666(2)$ & $0.035(2)$ & $0.033(2)$ & $0.034(2)$ & $-0.002(1)$ & $0.007(1)$ & $0.001(1)$ \\
\hline$C(11)$ & $4 e$ & $0.9377(4)$ & $-0.5529(3)$ & $-0.3163(2)$ & $0.032(2)$ & $0.052(2)$ & $0.042(2)$ & $0.004(2)$ & $0.011(1)$ & $0.003(2)$ \\
\hline$C(12)$ & $4 e$ & $1.0303(4)$ & $-0.4747(3)$ & $-0.2845(2)$ & $0.029(2)$ & $0.065(2)$ & $0.052(2)$ & $-0.011(2)$ & $0.010(2)$ & $0.003(2)$ \\
\hline$C(13)$ & $4 e$ & $0.9107(4)$ & $-0.4155(3)$ & $-0.2617(2)$ & $0.034(2)$ & $0.047(2)$ & $0.041(2)$ & $-0.014(2)$ & $0.003(1)$ & $0.002(2)$ \\
\hline$C(21)$ & $4 e$ & $0.3163(5)$ & $-0.4690(3)$ & $-0.4571(2)$ & $0.043(2)$ & $0.062(2)$ & $0.030(2)$ & $0.007(2)$ & $0.004(1)$ & $0.002(2)$ \\
\hline$C(22)$ & $4 e$ & $0.2744(5)$ & $-0.3756(3)$ & $-0.4512(2)$ & $0.053(2)$ & $0.063(3)$ & $0.049(2)$ & $0.021(2)$ & $0.003(2)$ & $0.014(2)$ \\
\hline$C(23)$ & $4 e$ & $0.3702(5)$ & $-0.3459(3)$ & $-0.3776(2)$ & $0.047(2)$ & $0.040(2)$ & $0.052(2)$ & $0.010(2)$ & $0.013(2)$ & $0.009(2)$ \\
\hline$C(31)$ & $4 e$ & $0.4033(4)$ & $-0.6105(2)$ & $-0.1712(2)$ & $0.044(2)$ & $0.040(2)$ & $0.045(2)$ & $0.002(2)$ & $0.015(1)$ & $0.005(2)$ \\
\hline$C(32)$ & $4 e$ & $0.4031(5)$ & $-0.5454(3)$ & $-0.1083(2)$ & $0.064(2)$ & $0.055(2)$ & $0.040(2)$ & $0.005(2)$ & $0.023(2)$ & $0.006(2)$ \\
\hline$C(33)$ & $4 e$ & $0.4724(4)$ & $-0.4663(2)$ & $-0.1348(2)$ & $0.050(2)$ & $0.045(2)$ & $0.029(2)$ & $0.005(2)$ & $0.008(2)$ & $-0.005(2)$ \\
\hline$C(40)$ & $4 e$ & $0.2561(4)$ & $-0.7358(2)$ & $-0.4066(2)$ & $0.033(2)$ & $0.030(2)$ & $0.043(2)$ & $0.002(1)$ & $0.005(1)$ & $-0.001(1)$ \\
\hline$C(41)$ & $4 e$ & $0.1312(4)$ & $-0.7797(2)$ & $-0.4767(2)$ & $0.032(2)$ & $0.034(2)$ & $0.038(2)$ & $0.001(1)$ & $0.003(1)$ & $-0.001(1)$ \\
\hline$C(42)$ & $4 e$ & $0.1354(4)$ & $-0.7573(3)$ & $-0.5578(2)$ & $0.041(2)$ & $0.049(2)$ & $0.040(2)$ & $-0.007(2)$ & $0.010(1)$ & $-0.002(2)$ \\
\hline$C(43)$ & $4 e$ & $0.0189(5)$ & $-0.7973(3)$ & $-0.6215(2)$ & $0.052(2)$ & $0.065(2)$ & $0.037(2)$ & $-0.001(2)$ & $0.005(2)$ & $-0.006(2)$ \\
\hline$C(44)$ & $4 e$ & $-0.1004(5)$ & $-0.8612(3)$ & $-0.6049(2)$ & $0.051(2)$ & $0.065(3)$ & $0.046(2)$ & $-0.010(2)$ & $-0.005(2)$ & $-0.012(2)$ \\
\hline$C(45)$ & $4 e$ & $-0.1061(6)$ & $-0.8843(4)$ & $-0.5249(3)$ & $0.080(3)$ & $0.096(4)$ & $0.062(3)$ & $-0.056(3)$ & $-0.002(2)$ & $0.003(3)$ \\
\hline$C(46)$ & $4 e$ & $0.0096(6)$ & $-0.8431(3)$ & $-0.4608(2)$ & $0.076(3)$ & $0.086(3)$ & $0.041(2)$ & $-0.041(2)$ & $-0.002(2)$ & $0.010(2)$ \\
\hline
\end{tabular}

Acknowledgment. The Australian Research Council is thanked for support.

\section{References}

1. Van Langenberg, K. A.: Ph.D. Thesis, Monash University, Clayton, Victoria, Australia 1999.

2. Cvetkovic, M.; Batten, S. R.; Moubaraki, B.; Murray, K. S.; Spiccia, L.: Copper(I) tris(pyrazolyl)methane complexes and their reactivity towards dioxygen Inorg. Chim. Acta 324 (2001) 131-140.

3. Kitajima, N.; Moro-Oka, Y.: Copper dioxygen complexes. Inorganic and bioinorganic perspectives. Chem. Rev. 94 (1994) 737-757.

4. Tolman, W. B.: Making and breaking the dioxygen $\mathrm{O}-\mathrm{O}$ bond: new insights from studies of synthetic copper complexes. Acc. Chem. Res. 30 (1997) 227-237.

5. Altomare, G.; Cascarano, G.; Giacovazzo, C.; Guagliardi, A.; Burla, M. C.; Polidori, G.; Camalli, M.: SIR92 - a program for automatic solution of crystal structures by direct methods. J. Appl. Crystallogr. 27 (1994) 435.
6. teXsan: Single Crystal Structure Analysis Software. Version 1.04. Molecular Structure Corporation. The Woodlands, TX, USA 1997.

7. Sheldrick, G. M.: SHELXL-97. Program for crystal structure refinement. University of Göttingen, Germany 1997.

8. Spek, T.: PLATON, A Multipurpose Crystallographic Tool, Utrecht University, Utrecht, The Netherlands 2000.

9. Walker, N.; Stuart, D.: An empirical method for corrrecting diffractometer data for absorption effects. Acta Crystallogr. A39 (1983) 158-166.

10. Johnson, C. K.: ORTEPII. Report ORNL-5138, Oak Ridge National Laboratory, Tennessee, USA 1976. 